\title{
Internet of People and situated computing
}

\author{
Ching-Hsien Hsu ${ }^{1}$
}

(C) Springer-Verlag London 2016

\section{Introduction}

The new term Internet of People (IoP) has been predicted as the next wave of information and communications technologies (ICT) after the widely accepted smartphones market. Some has estimated that more than 55 billion of IoP devices could be sold in 2025 and our future life might be surrounded by a lot of IoP devices. The success of IoP will heavily rely on the virtuous cycle of innovations from cloud computing, to broadband networks, to big data, and to smart but lightweight devices. Notwithstanding, there are many challenges to meet, in order to have the IoP smarter. The most complicated and at the same time challenging paradigm is the situated computing that highly relies on users' physical contexts and objects' interactions to provide services, being projected as a future growth area in both academia and industry.

This special issue aims at presenting the current state-ofthe-art research and future trends on various aspects of IoP technologies. It combines the emerging mobile communications and IoP paradigms, in a common research ground, in order to present various research concepts. The major subjects of this special issue cover methodologies, modelling, analysis of human behavior and activities, newly introduced communication technologies and applications, as well as computational intelligence for ambient assisted living.

Papers selected for this special issue represent recent progresses in the field, including works on M2M

Ching-Hsien Hsu

chh@chu.edu.tw

1 Department of Computer Science and Information Engineering, Chung Hua University, Hsinchu, Taiwan communication, information security, mobile social networks, machine learning. All of these papers not only provide novel ideas and state-of-the-art techniques in the field, but also stimulate future research in the sustainable environment.

\section{Communication and information security}

Internet of Things (IoT) has been considered as one of the most promising technologies over the next decade. One of the basic requirements of IoT is the global communication connectivity between smart objects. LTE-A has been considered as the main communication channel for connecting devices. The paper by Ren-Hung Hwang, Chi-Fu Huang, Huang-Wei Lin, Jang-Jiin Wu entitled "Uplink Access Control for Machine-Type Communications in LTE-A Networks" proposed an adaptive random access channel resource allocation (ARRA) scheme which combines the RACH resource allocation, access class barring, and priority device setting. The proposed ARRA provides a complete flow to handle the large number of the machinetype communication (MTC) devices accessing the LTE-A network. In addition, both the human-type communication (HTC) and MTC devices were considered, as well as separating the devices into different service types. Different priorities were assigned to different types of devices to meet their QoS requirements. Simulation results show that the ARRA guarantees the access success probability of the Class A devices.

The emergence of smart manufacturing drives demands for M2M communication. The paper by Tao Lei, Shangguang Wang, Jinglin Li, Fangchun Yang entitled "AOM: Adaptive Mobile Data Traffic Offloading for M2M Networks" focuses on how to adaptively offload data traffic for 
cellular M2M networks. The authors propose an adaptive mobile data traffic offloading model (AOM), which is able to dynamically decide whether to adopt opportunistic communications or communicate via cellular networks. This work introduces traffic offloading rate and local resource consumption rate and analyses them based on continuous-time Markov chain. Theoretical proof and extensive simulations demonstrate that the proposed model is accurate and effective and can adaptively offload data traffic in cellular M2M networks.

With the popularity of mobile phones with Android platform, Android platform-based individual privacy information protection has been paid significant attention. The paper by Weizhe Zhang, Xiong Li, Naixue Xiong, Athanasios V. Vasilakos entitled "Android Platform-based Individual Privacy Information Protection System" proposed a mobile terminal system with the feature of selfprotection. The proposed architecture uses SMS for remote control of mobile phones and provides comprehensive individual information protection for users. The main innovation of this work is that the system is free from the support of the server and it can provide individual information protection for users by the most basic SMS function. Moreover, the protection mechanism of the redundancy process trusted number mechanism and SIM card detection mechanism are also novel techniques of this system.

\section{Theory and methodologies}

With the rapid proliferation of wireless portable devices with Bluetooth or Wi-Fi, opportunistic mobile social networks begin to emerge. The paper by Huan Zhou, Linping Tong, Shouzhi Xu, Chungming Huang, Jialu Fan entitled "Predicting Temporal Centrality in Opportunistic Mobile Social Networks Based on Social Behaviour of People" focuses on predicting nodes' future centrality from the temporal perspective using real mobility traces in opportunistic mobile social networks. Three important centrality metrics, namely betweenness, closeness, and degree centrality, are considered. Through real trace-driven simulations, this research finds that nodes' future centrality is highly predictable due to natural social behaviour of people. Based on the observations in the simulation, the authors design several reasonable prediction methods to predict nodes' future temporal centrality. Extensive real trace-driven simulations were conducted in this work.

The future Internet is expected to connect billions of people, things, and services having the potential to deliver a new set of applications by deriving new insights from the data generated from these diverse data sources. This highly interconnected global network brings new types of challenges in analysing and making sense of Data. This is why machine learning is expected to be a crucial technology in the future, in making sense of data, in improving business and decision making, and in so doing providing the potential to solve a wide range of problems in healthcare, telecommunications, urban computing, and others. The paper by Haytham Assem, Lei Xu, Teodora Sandra Buda, Declan O'Sullivan entitled "Machine Learning as a Service for Enabling Internet of Things and People" introduces the TCDC (train, compare, decide, and change) approach, which can be thought as a "Machine Learning as a Service" approach, to aid machine-learning researchers and practitioners to choose the optimum machine-learning model to use for achieving the best trade-off between accuracy and interpretability, computational complexity, and ease of implementation. The proposed approach tries to take into attention the trade-off between predictive performance and interpretability, complexity, and ease of implementation.

\section{Tools and applications}

Due to the growing popularity of Internet of People (IoP) and its impacts on human activity, it has quickly become an important research field and hot subject. Since one purpose of IoP is to connect people to people, personal live video delivery that has been popular recently can also be regarded as an important application of IoP. The paper by Shiuan-Tung Chen, Hsin-Ta Chiao, Shih-Ying Chang, Hung-Min Sun entitled "Personal Video Delivery with Rapid Raptor Code Decoder" proposes a new method to accelerate raptor code decoding based on some pre-calculated data. The system architecture, the generation algorithm of group parameters, the algorithm switching thresholds, and the method to reconstruct the intermediate symbol set are presented in detail. For a raptor code application that can frequently use one or several fixed source block lengths, one could produce the corresponding group parameters in advance and use them to decode the received encoding symbols more efficiently. For personal live video delivery scenario, the simulation results show that the proposed technique has superior performance.

Scientific research works conducted by researchers spreading all over the world in every research field, which are hard to be tracked and quantified. The paper by Wei Liang, Zixian Lu, Qun Jin, Min Wu, Chunhua Hu entitled "Analysing of Research Patterns Based on a Temporal Tracking and Assessing Model" seeks to model researchers' scientific activities and quantify their outcome during their research career. A temporal tracking and assessing model is introduced to represent research development and quantified the scientific outcome for both an individual and 
a group along the time. Based on the proposed model, a research topic analysing approach is utilized to extract the topics covered by a research group for the research pattern analysing. The latent research pattern discovering approach is proposed to depict how a research group's research works contributed by its members are discovered and visualized. The effectiveness of the proposed approach was evaluated based on a real academic dataset.

\section{Conclusions}

All of the above papers address either technical issues in M2M communication or information security or propose novel application models in the various IoP and mobile social networks fields. They also trigger further related research and technology improvements in application of situated computing. Honourably, this special issue serves as a landmark source for education, information, and reference to professors, researchers, and graduate students interested in updating their knowledge about or active in Internet of Things, Internet of People, and novel application models for future information services and systems.

This special issue of Personal and Ubiquitous Computing covers different aspects of the problem, from both the theoretical and practical side. After a large open call, an international editorial committee selected six research papers. Each paper was reviewed by at least three reviewers.

The guest editor would like to express sincere gratitude to Dr./Prof. Peter Thomas (EiC, PUC) for giving the opportunity to prepare this special issue. In addition, we are deeply indebted to numerous reviewers for their professional effort, insight and hard work put into commenting on the selected articles that reflect the essence of this special issue. Last but not least, we are grateful to all authors for their contributions and for undertaking two-cycle revisions of their manuscripts, without which this special issue could not have been produced. 